\title{
Variáveis cardiovasculares durante e após a prática do VíDEO GAME ativo "Dance Dance Revolution" e televisão
}

\author{
Suliane Beatriz Rauber 1,2,3 \\ Ferdinando Oliveira Carvalho ${ }^{1,4,5}$ \\ lorrany Raquel Castro de Sousa ${ }^{1,3}$ \\ Rafaello Pinheiro Mazzoccante ${ }^{1,3}$ \\ Carolina Belfort Sousa Franco ${ }^{1,3}$ \\ Darlan Lopes de Farias ${ }^{1,2}$ \\ Maritza Alves de Sousa Coura ${ }^{1,4}$ \\ Herbert Gustavo Simões $1,3,4$ \\ Carmen Silvia Grubert Campbell ${ }^{1,3,4}$ \\ 1 Grupo de Estudos do Desempenho Humano e das Respostas Fisiológicas ao Exercício Físico,
Universidade Católica de Brasília, Taguatinga, DF, Brasil
${ }^{2}$ Laboratório de Educação Física e Saúde, Faculdade de Educação Física, Universidade Católica de
Brasília, Taguatinga, DF, Brasil
${ }^{3}$ Faculdade de Educação Física, Universidade Católica de Brasília, Taguatinga, DF, Brasil
${ }^{4}$ Programa de Pós-Graduação Stricto Sensu em Educação Física, Universidade Católica de Brasília,
Taguatinga, DF, Brasil
${ }^{5}$ Curso de Educação Física da Universidade Federal do Vale do São Francisco, Petrolina, PE, Brasil
}

Resumo: As doenças cardiovasculares (DCVs) estão entre as principais causas de morte no mundo e os processos patológicos associados com o seu desenvolvimento têm início na infância. O objetivo do estudo foi analisar e comparar as respostas de variáveis cardiovasculares durante e após a prática de vídeo game interativo e televisão. A amostra foi composta de oito crianças saudáveis $\left(9,8 \pm 0,5\right.$ anos; $\left.30,1 \pm 3,4 \mathrm{~kg} ; 133,3 \pm 13,6 \mathrm{~cm} ; 17,4 \pm 4,7 \mathrm{~kg} / \mathrm{m}^{2}\right)$. O estudo consistiu de três sessões das quais a 1를 destinou-se a avaliação antropométrica e familiarização (FAM) com os equipamentos e o laboratório, e as demais sessões foram realizadas em ordem randomizada, sendo 30 minutos de Dance Dance Revolution (DDR), nível iniciante; e 30 minutos de televisão (TV) em que dois desenhos infantis (Ben 10 e Bob Esponja) foram apresentados. Durante as sessões foram aferidas a freqüência cardíaca (FC), Pressão Arterial Sistólica (PAS) e Pressão Arterial Diastólica (PAD) aos 10 minutos de repouso sentado, bem como aos 10, 20 e 30min de atividade (DDR ou TV) e aos 2, 10, 20, 30 e 40 min após finalização das atividades. Para a análise estatística foi verificada a distribuição normal dos dados pelo teste de KolgomorovSmirnov, ANOVA one-way, com Post Hoc de Scheffé para comparação entre sessões e ANOVA para medidas repetidas na comparação entre os momentos de cada sessão que foram testados. O nível de significância adotado foi p $\leq 0,05$ (ESTATíSTICA 6.0). Aumento significativo dos valores de FC, PAS e PAM durante o DDR (30 min: $110 \mathrm{bpm}, 116 \mathrm{e} 89 \mathrm{mmHg}$ ) foram observados quando comparados com a TV (30 min: 86 bpm, 102 e $70 \mathrm{mmHg})$ respectivamente. Foi observada uma tendência de queda da FC e PAS aos 40 min de recuperação após sessão DDR, bem como uma tendência de elevação da FC, PAS, PAD e PAM após TV quando comparadas ao repouso, porém estas não foram significantes $(p>0,05)$. Concluímos que o vídeo game interativo DDR pode ser uma alternativa interessante para aumentar o nível de atividade física, podendo trazer benefícios cardiovasculares pósexercício. Caso estes valores de PAS se mantenham inferiores nessas crianças ao longo do dia, podemos considerar o uso desse instrumento na proteção cardiovascular durante a infância, podendo impactar positivamente na atenuação de eventos cardiovasculares na idade adulta futura, o que seria muito importante para a saúde pública.

Palavras-chave: Crianças. Respostas cardiovasculares e vídeo game ativo.

\section{Cardiovascular responses during and after active VIDEO GAME and television}

\begin{abstract}
Cardiovascular diseases (CVDs) are the leading cause of death worldwide and the pathological processes associated with their development in the early childhood. The objective of this study was to investigate the cardiovascular responses during and after the practice of video game interactive and television. The sample consisted of eight healthy children $\left(9.8 \pm 0.5\right.$ years, $\left.30.1 \pm 3.4 \mathrm{~kg}, 133.3 \mathrm{~cm} \pm 13.6,17.4 \pm 4.7 \mathrm{~kg} / \mathrm{m}^{2}\right)$. The study consisted of three sessions, where the 1st was FAM - for anthropometric measurement and procedures familiarization, and the other sessions, were performed in randomized order; DDR - Dance Dance Revolution: the volunteers realized 30 min in the beginner level; TV 30 min watching a children's cartoon (Ben 10 and Sponge Bob). The heart rate (HR), systolic (SBP) and diastolic (DBP) blood pressure were measured at rest and during the 10th, 20th and 30th minutes of activity and recovery in the 2nd 10th, 20th, 30th and 40th min. Statistical analysis was checked for normal distribution of data by Kolgomorov-Smirnov test, ANOVA, one-way MANOVA with post hoc Scheffé for comparison between sessions, ANOVA repeated measures, comparing the times of each session. The significance level was $p \leq 0.05$ (ESTATÍSTICA 6.0). The results show a significant rise in HR, SBP and MAP during the DDR (30min: $110 \mathrm{bpm}, 116.3$ and $89.6 \mathrm{mmHg}$ ) compared to the TV (30min: $86.7 \mathrm{bpm}, 102 \mathrm{mmHg}$ and $70.3 \mathrm{mmHg}$ ). There was a downward trend in HR and SBP at 40 min of recovery after session DDR, as well as an upward trend in HR, SBP, DBP, and MAP after TV when compared to the rest, but these were not significant $(p>0,05)$. We conclude that video game is an interesting alternative of active playing, which might bring cardiovascular benefits. If these BP values be maintained throughout the day during childhood, we may expect a better health during adulthood what, in turn, may be important for public health.
\end{abstract}

Keywords: Children. Cardiovascular responses and video game active. 


\section{Introdução}

As doenças cardiovasculares (DCVs) são a principal causa de morte no mundo e os processos patológicos associados com o seu desenvolvimento geralmente têm início na infância. A hipertensão arterial (HA), um dos principais fatores de risco para DCVs, já tem sido relatado na população pediátrica (LUNA e SPIOTA, 2006). A relação entre a atividade física $(\mathrm{AF})$ e a pressão arterial (PA) em adultos está bem estabelecida, mas pouco são os estudos conduzidos em crianças. Estudos populacionais apontam uma relação entre um baixo nível de AF e valores de pressão arterial mais elevados em adolescentes (LEARY et al., 2008) e uma correlação positiva entre 0 aumento da circunferência da cintura e a PA nessa população (CARVALHO et al., 2012).

O estilo de vida sedentário tem contribuído para o aumento da incidência de obesidade e doenças crônicas como a HA na população pediátrica (BAKER, OLSEN, SORENSEN, 2007; CARLETTI, RODRIGUES, PEREZ, VASSALLO, 2008; SYME et al., 2009; OBARZANEK et al., 2010). O hábito sedentário de despender tempo elevado na frente da televisão ou de equipamentos eletroeletrônicos é bastante comum entre crianças e adolescentes (PELEGRINI, SILVA, PETROSKI, 2008; MARTINEZ-GOMEZ, TUCKER, HEELAN, WELK, EISENMANN., 2009; CORSO et al., 2012). No entanto, apesar de bastante conhecidos os benefícios oferecidos pela $A F$, muitas crianças e jovens em idade escolar não atendem as recomendações básicas (TROIANO et al., 2008; LEARY et al., 2008), preconizadas pela Organização Mundial da Saúde (OMS, 2010) de acumular pelo menos 60 minutos de atividade física de intensidade moderada a vigorosa para garantir um desenvolvimento saudável.

Ainda nessa direção, estratégias para elevar a prática de atividade física das crianças e adolescentes têm sido implementadas por meio de intervenções de base escolar (JAGO e BARANOWSKI, 2004). No entanto, a luta contra o sedentarismo deve ser abordada em todos os espaços de convivência da criança, incluindo o ambiente doméstico, já que é neste ambiente que as crianças gastam uma parcela significativa do seu dia em comportamentos sedentários, como ver televisão ou jogar vídeo game (ROBINSON, 1999; ATKIN, GORELY, BIDDLE, MARSHALL, CAMERON, 2008).

Jogos de vídeo game ativos foram desenvolvidos visando minimizar o problema da inatividade física infantil em ambiente doméstico. Estudos recentes indicam que esse tipo de atividade pode ter efeitos metabólicos benéficos por meio do aumento do gasto energético (WANG e PERRY, 2006). O vídeo game ativo é uma opção de atividade que aumenta o consumo de oxigênio, gasto calórico e a freqüência cardíaca, sendo considerado uma ferramenta interessante na adesão à prática de AF (MARK, RHODES, DARREN, WARBURTON, 2008). Contudo, ainda existe uma carência de estudos na literatura envolvendo respostas cardiovasculares durante e após a prática desses novos modelos de jogos, sobretudo na população pediátrica. Assim, o objetivo desse estudo foi verificar e comparar a resposta das variáveis cardiovasculares durante e após a prática de vídeo game interativo "Dance Dance Revolution" e televisão.

\section{Materiais e Métodos}

Participaram do estudo oito crianças com idade entre 9 e 10 anos, ambos os sexos (quatro meninas e quatro meninos), raça negra e branca, estudantes do ensino fundamental participantes de um projeto social da UCB. Para participar do estudo os voluntários não podiam ter tido contato prévio com o Dance Dance Revolution (DDR). O estudo foi aprovado pelo comitê de ética em pesquisa em seres humanos da Universidade Católica de Brasília - UCB (147/2009) e só teve início após assinatura do termo de consentimento livre e esclarecido pelo responsável pela criança.

As coletas foram realizadas no Laboratório de Estudos em Educação Física e Saúde (LEEFS) da Universidade Católica de Brasília, entre 8 e $11 \mathrm{~h}$ da manhã, tendo cada sessão uma duração de $80 \mathrm{~min}$, divididos em: $10 \mathrm{~min}$ de repouso, 30 min de atividade e 40 min de recuperação. Para a realização deste estudo foram necessárias três visitas, sendo a primeira de familiarização com os equipamentos, a pesquisadora e os procedimentos do estudo; e as outras duas sessões foram realizadas em ordem randomizada - televisão (TV) e DDR.

Sessão para avaliação antropométrica e familiarização (FAM) - A massa corporal (MC) 
$(\mathrm{kg})$ foi mensurada em uma balança eletrônica (Tech 05, China) e a estatura $(\mathrm{cm})$ em um estadiômetro (Cardiomed, Brasil). Para calcular o Índice de Massa Corporal (IMC) foi dividida a massa corporal em $\mathrm{kg}$ pela estatura em metros ao quadrado $\left(\mathrm{IMC}=\mathrm{MC}(\mathrm{kg}) / \mathrm{E}^{2}(\mathrm{~m})\right)$. Foi utilizado um adipômetro (Lange Skinfold Caliper - EUA) para mensuração das dobras cutâneas, triciptal e subescapular $(\mathrm{mm})$, cujos valores foram empregados para o cálculo da porcentagem de gordura (\%G) de acordo com o proposto por SLAUGHTER et al. (1988), sendo:

Para meninas negras e brancas - 7 a 17 anos:

$\% \mathrm{G}=1,33(\mathrm{TR}+\mathrm{SE})-0,013(\mathrm{TR}+\mathrm{SE})^{2}-2,5$

Para meninos pré-puberes negros:

$\% \mathrm{G}=1,21$ (TR+SE) - 0,008 (TR+SE) $)^{2}-3,5$

Para meninos pré-puberes brancos:

$\% \mathrm{G}=1,21$ (TR+SE) - 0,008 (TR+SE) $)^{2}-1,7$

Após estas mensurações, foi feita a familiarização do voluntário com o uso dos equipamentos (esfigmomanômetro e estetoscópio), bem como foram fornecidas instruções sobre o DDR, em que o voluntário recebia as orientações sobre o jogo, sobre como jogar, e logo após, era convidado a realizar algumas tentativas no nível iniciante proposto pelo fabricante do equipamento. Adotamos como critério para familiarização todas as crianças jogarem entre 10 e 15 vezes. As crianças voluntárias nunca tinham tido contato prévio com este tipo de brinquedo interativo anteriormente à familiarização feita neste estudo.

Sessão Vídeo Game (DDR) - O voluntário participou ativamente, durante $30 \mathrm{~min}$, de um jogo no vídeo game interativo (Dance Dance Revolution - DDR), instalado em um notebook da marca Nextera (15 polegadas). O voluntário escolhia as músicas dentre uma seleção de mesmo ritmo, feita anteriormente para todos os voluntários, com baixo nível de dificuldade (nível básico).

Sessão Televisão (TV) - Os voluntários foram convidados a assistir dois desenhos conhecidos (Bob Esponja e Ben 10), com duração de $15 \mathrm{~min}$ cada, totalizando $30 \mathrm{~min}$, em uma sala silenciosa. Os filmes foram apresentados no mesmo notebook da marca Nextera (15 polegadas), em que o DDR foi apresentado.

\section{Variáveis Cardiovasculares}

A pressão arterial sistólica (PAS), diastólica (PAD) e a freqüência cardíaca ( $F C)$ foram mensuradas durante cada sessão. Aos cinco e $10 \mathrm{~min}$ de repouso sentado présessão, aos 10,20 e $30 \mathrm{~min}$ durante as sessões e imediatamente após as sessões; e aos 2, 10, 20, 30 e 40 min após cada sessão. A partir da PAS e PAD foi calculada a pressão arterial média (PAM). A PA foi aferida por uma única avaliadora experiente, no braço direito do voluntário, pelo método auscultatório, com o voluntário sentado em cadeira confortável com encosto e os joelhos flexionados a $90^{\circ}$, segundo as recomendações do Fourth Task Force (1996), utilizando-se esfigmomanômetro pediátrico (BD, Brasil), de manguito adequado à circunferência do braço da criança $e$ estetoscópio infantil (Missouri, Brasil). A FC foi obtida por um freqüencímetro (Polar FS1, Finlândia).

\section{Análise Estatística}

A normalidade dos dados foi confirmada utilizando o teste de Kolgomorov-Smirnov. Para apresentação dos valores de média, desviopadrão, mínimo e máximo foi utilizada a estatística descritiva. Para comparação entre as sessões (TV e DDR) ANOVA-MANOVA one-way, e quando encontradas diferenças foi empregado Post Hoc de Scheffé para localizá-las. Foi utilizada ANOVA para medidas repetidas na comparação entre os momentos dentro de cada sessão. O nível de significância adotado foi de $\mathrm{P} \leq 0,05$ e o pacote estatístico para análise foi o ESTATÍSTICA 6.0.

\section{Resultados}

A tabela 1 apresenta os valores descritivos da amostra. A amostra foi composta por crianças com idade entre nove e 10 anos, média de massa corporal, estatura, IMC e \% G próximo do percentil 50 (WHO). Os valores médios de repouso para PAS $(101 \pm 2,5 \mathrm{mmHg})$ e PAD $(65,9$ $\pm 1,4 \mathrm{mmHg}$ ) corresponderam aos percentis $50 \mathrm{e}$ 60 respectivamente, segundo as tabelas do National Heart, Lung and Blood Institute (HHLBI) (2004). Apesar de se conhecer as diferenças entre meninos e meninas em algumas variáveis, como \%G e FC, optou-se por não realizar análise entre grupos separados por sexo em decorrência de um número pequeno da amostra, o que pode justificar o desvio padrão elevado em algumas variáveis. 
Tabela 1. Valores descritivos da amostra $(n=8)$.

\begin{tabular}{lc}
\hline \multicolumn{1}{c}{ VARIÁVEL } & MÉDIA \pm DP \\
\hline Idade (anos) & $9,8 \pm 0,5$ \\
Estatura (cm) & $133,3 \pm 13,6$ \\
Massa Corporal $(\mathbf{k g})$ & $30,1 \pm 3,4$ \\
$\%$ G & $16,7 \pm 5,8$ \\
IMC (kg/m $\left.{ }^{2}\right)$ & $17,4 \pm 4,7$ \\
CB $(\mathbf{c m})$ & $18,8 \pm 1,1$ \\
CC $(\mathbf{c m})$ & $57,3 \pm 3,2$ \\
PAS repouso (mmHg) & $101,0 \pm 2,5$ \\
PAD repouso (mmHg) & $65,9 \pm 1,4$ \\
FC repouso (bpm) & $84,7 \pm 1,3$ \\
\hline
\end{tabular}

Nota: $\% G$ - Porcentagem de gordura estimada pelas equações propostas por SLAUGHTER (1988); IMC - Índice de Massa Corpórea; CB - Circunferência do Braço; CC - Circunferência da Cintura. PAS Pressão Arterial Sistólica; PAD - Pressão Arterial Diastólica; FC - Frequência Cardíaca.
$\mathrm{Na}$ tabela 2 estão apresentados os valores de FC, PAS, PAD e PAM em cada momento das sessões. Foram observados valores superiores aos 10,20 e $30 \mathrm{~min}$ do DDR quando comparados com a sessão TV nas seguintes variáveis: FC (DDR-105; 108,5; e 110,2 bpm vs TV-87,2; 87,7; e 86,7 bpm, respectivamente), PAS (DDR - 113,8; 116,8; e $116,9 \mathrm{mmHg}$ vs TV- 103,3; 101,1; e $102,8 \mathrm{mmHg}$, respectivamente) e PAM (DDR-86,1; 88,8; e 89,6 $\mathrm{mmHg}$ vs TV - 80,6; 80,4; e 81,1 mmHg, respectivamente). Não foi observada redução significativa da PA e da FC após sessão DDR, porém enquanto a maior parte destes valores tende a cair após esta sessão, tendem a se elevar após TV quando comparados com o repouso intra-sessão.

Tabela 2. Valores de FC, PAS, PAD e PAM (Média \pm DP) durante e após as sessões TV e DDR $(n=8)$.

\begin{tabular}{|c|c|c|c|c|c|c|c|c|}
\hline \multirow[b]{2}{*}{ TEMPO } & \multicolumn{4}{|c|}{ TV } & \multicolumn{4}{|c|}{ DDR } \\
\hline & FC & PAS & PAD & PAM & FC & PAS & PAD & PAM \\
\hline Repouso & $82,9 \pm 6,0$ & $102,0 \pm 6,9$ & $67,0 \pm 3,9$ & $78,7 \pm 3,7$ & $87,8 \pm 8,1$ & $103,5 \pm 4,7$ & $65,2 \pm 5,5$ & $78,3 \pm 4,5$ \\
\hline $10 \mathrm{~min}$ & $87,2 \pm 3,7$ & $103,3 \pm 4,7$ & $69,3 \pm 5,7$ & $80,6 \pm 3,9$ & $105,0 \pm 10,2^{\#}$ & $113,8 \pm 5,8^{\#}$ & $70,4 \pm 6,1$ & $86,1 \pm 4,6^{\#}$ \\
\hline $20 \mathrm{~min}$ & $87,7 \pm 4,5$ & $101,1 \pm 7,9$ & $70,0 \pm 6,0$ & $80,4 \pm 4,1$ & $108,5 \pm 13,2^{\#}$ & $116,8 \pm 7,2^{\#}$ & $72,8 \pm 5,9$ & $88,8 \pm 4,7^{\#}$ \\
\hline $30 \mathrm{~min}$ & $86,7 \pm 5,0$ & $102,8 \pm 7,2$ & $70,3 \pm 6,5$ & $81,1 \pm 4,9$ & $110,2 \pm 12,4^{\#}$ & $116,9 \pm 6,9^{\#}$ & $74,0 \pm 6,8$ & $89,6 \pm 4,6^{\#}$ \\
\hline R2 min & $88,1 \pm 5,3$ & $101,5 \pm 8,4$ & $68,8 \pm 6,7$ & $79,7 \pm 5,9$ & $94,5 \pm 11,5$ & $107,3 \pm 4,3$ & $70,4 \pm 6,3$ & $83,3 \pm 4,3$ \\
\hline R10 min & $89,0 \pm 5,4$ & $103,3 \pm 7,6$ & $69,0 \pm 5,0$ & $80,4 \pm 5,0$ & $92,2 \pm 10,7$ & $103,5 \pm 3,7$ & $68,8 \pm 6,9$ & $80,8 \pm 4,8$ \\
\hline R20 min & $91,8 \pm 8,0$ & $103,0 \pm 5,8$ & $69,5 \pm 4,8$ & $80,7 \pm 4,4$ & $89,0 \pm 11,1$ & $101,8 \pm 3,5$ & $66,4 \pm 7,3$ & $78,9 \pm 5,2$ \\
\hline $\mathrm{R} 30 \mathrm{~min}$ & $92,6 \pm 9,3$ & $103,8 \pm 7,3$ & $70,3 \pm 4,7$ & $81,4 \pm 4,2$ & $88,0 \pm 9,7$ & $101,0 \pm 4,3$ & $66,0 \pm 6,7$ & $78,3 \pm 4,7$ \\
\hline R40 min & $93,7 \pm 9,7$ & $103,5 \pm 5,9$ & $70,8 \pm 4,8$ & $81,7 \pm 3,9$ & $84,8 \pm 10,3$ & $99,5 \pm 3,7$ & $65,4 \pm 6,9$ & $77,3 \pm 4,8$ \\
\hline
\end{tabular}

Nota: R - Recuperação; TV - Televisão; DDR - dance dance revolution; FC - Freqüência Cardíaca; PAS - Pressão Arterial

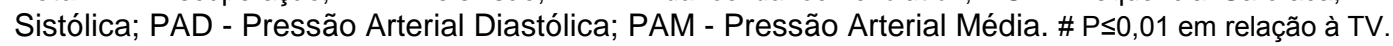

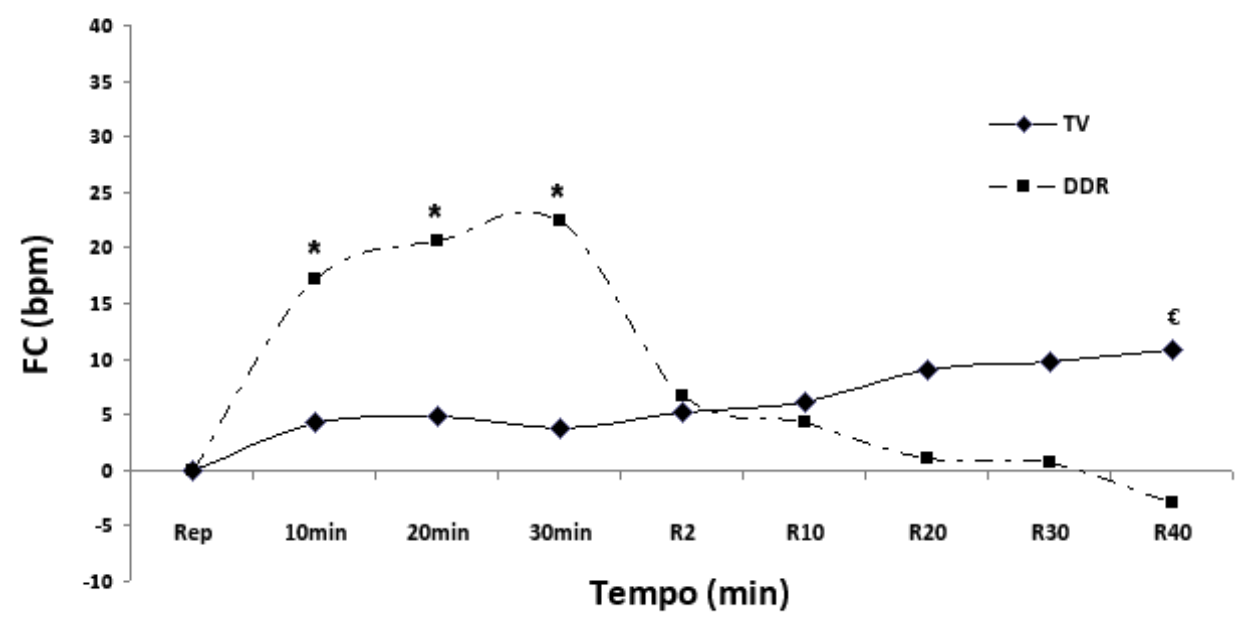

Figura 1. Valores de delta da FC (bpm) durante os 30 min de sessão (10, 20 e 30 min) e durante os minutos 2, 10, 20, 30 e 40 de recuperação (R2, R10, R20, R30 e R40) após as sessões de TV e DDR (Dance Dance Revolution); Rep - repouso; TV ( $F=3,33$ e $p \leq 0,03) ; \operatorname{DDR}(F=32,28$ e $p \leq 0,01)$; * $p \leq 0,01$ em relação ao repouso; $€ p \leq 0,05$ em relação ao repouso.

A figura 1 apresenta os deltas dos valores de cada variável em cada momento em relação ao repouso. A FC aumentou em média 15bpm a mais durante a sessão de DDR quando 
comparada com a sessão TV. Quando comparada a $\mathrm{FC}$ no repouso com a da R40 na sessão DDR, não foi observada redução significativa $(-3 b p m ; p>0,05)$, enquanto que na sessão TV, neste mesmo momento, observou-se um aumento significativo de 13\% (+11bpm; $p<0,05)$, quando comparado com o repouso.

$\mathrm{Na}$ figura 2 é possível observar a tendência de redução da PAS durante a recuperação após sessão DDR em relação aos valores de repouso, a partir dos $10 \mathrm{~min}$ de recuperação. Foi observada diferença significativa entre os deltas de variação da PAS aos 2 e 40 min de recuperação. Comparando-se a PAS na R40 com o repouso, notamos uma queda de $4 \mathrm{mmHg}$ após DDR, porém não houve diferença significativa.

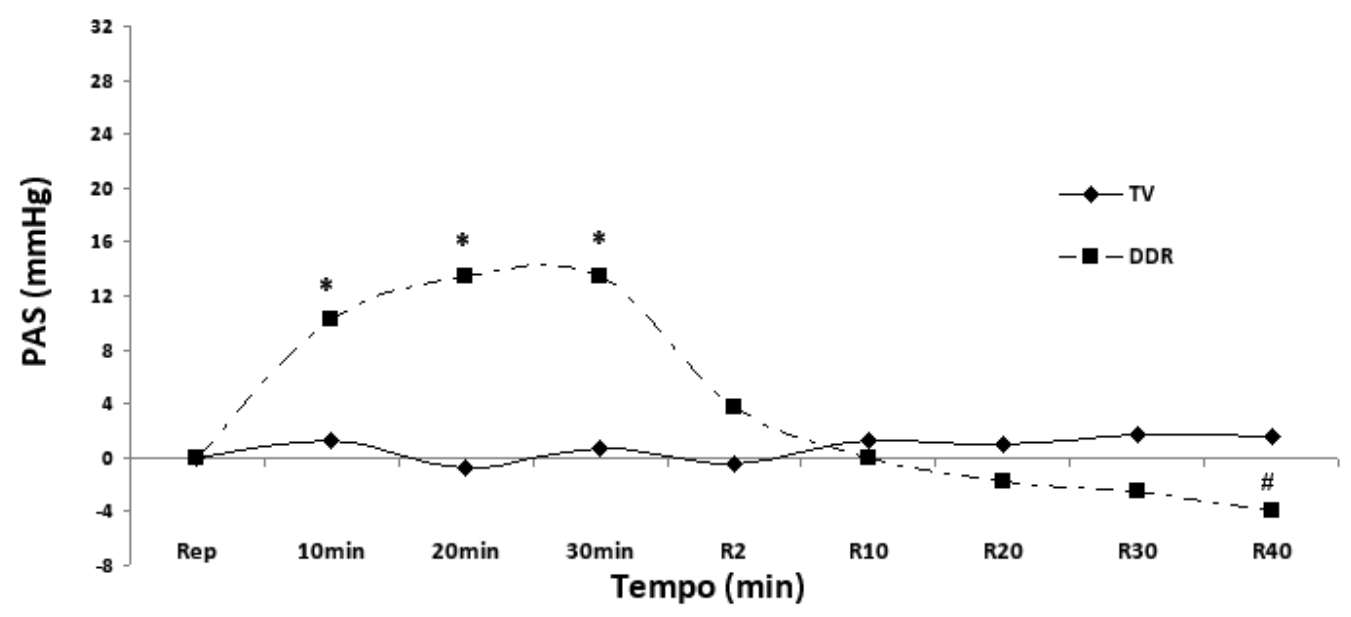

Figura 2. Valores de delta da PAS (mmHg) durante os 30 min de sessão $(10,20$ e $30 \mathrm{~min})$ e durante os minutos 2, 10, 20, 30 e 40 de recuperação (R2, R10, R20, R30 e R40) após as sessões de TV e DDR (Dance Dance Revolution); Rep - Repouso; TV ( $F=1,02$ e $p \leq 0,43)$; DDR ( $F=28,68$ e $p \leq 0,01)$; ${ }^{*} p \leq 0,01$ em relação ao repouso; \# p $\leq 0,01$ em relação ao $\mathrm{R} 2$.

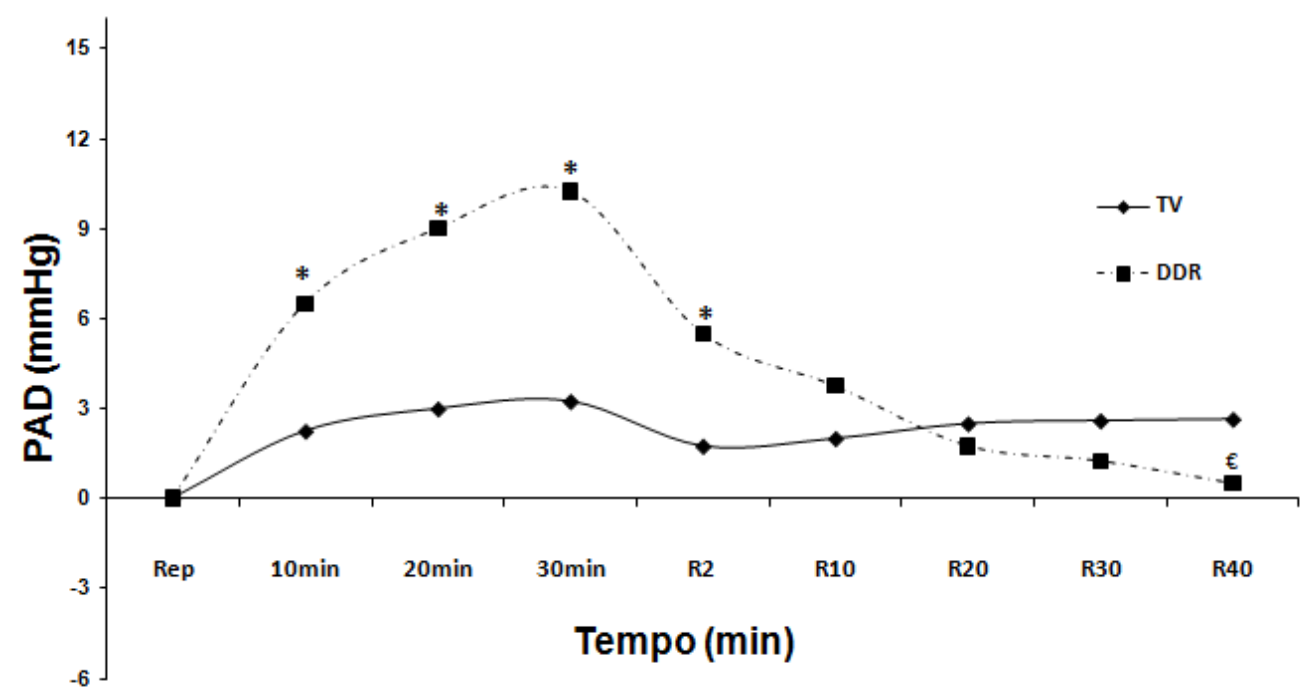

Figura 3. Valores de delta da PAS (mmHg) durante os 30 min de sessão (10, 20 e $30 \mathrm{~min})$ e durante os minutos 2, 10, 20, 30 e 40 de recuperação (R2, R10, R20, R30 e R40) após as sessões de TV e DDR (Dance Dance Revolution); Rep - Repouso; TV ( $F=1,02$ e $p \leq 0,43)$; DDR ( $F=28,68$ e $p \leq 0,01)$. ${ }^{*} p \leq 0,01$ em relação ao repouso; $€ \mathrm{p} \leq 0,05$ em relação ao $\mathrm{R} 2$;

Na sessão DDR, foi observado um aumento significativo da PAD nos momentos 10, 20,30min durante a sessão; e no momento $\mathrm{R} 2(+5,+7,+9$ e $+5 \mathrm{mmHg}$, respectivamente), em relação ao 
repouso da mesma sessão (Figura 3). Foi observada diferença significativa da PAD entre os momentos R2 e R40 da sessão DDR (Figura 3).

A figura 4 apresenta as diferenças na PAM entre os diferentes momentos de cada sessão. Valores de PAM encontraram-se significativamente elevados aos 10,20 e $30 \mathrm{~min}$ durante a sessão, e no momento R2 $(86,1 ; 88,8$; 89,6 ; e $83,3 \mathrm{mmHg}$ respectivamente) da sessão DDR, quando comparados ao valor de repouso pré sessão $(78,3 \mathrm{mmHg})$. Quando comparados os valores de PAM durante a recuperação, foi verificada diferença significativa nos momentos $\mathrm{R} 30$ e $\mathrm{R} 40$ (78,2 e 77,3 mmHg respectivamente) na sessão DDR em relação ao momento R2, sendo estes valores muito próximos ao de repouso $(78,3 \mathrm{mmHg})$. Nenhuma diferença significativa foi verificada entre os valores de PAM obtidos nos diferentes momentos da sessão TV. Porém observa-se uma curva ascendente da PAM, atingindo uma média aproximada de +3 $\mathrm{mmHg}$ em relação ao repouso no momento $\mathrm{R} 40$.

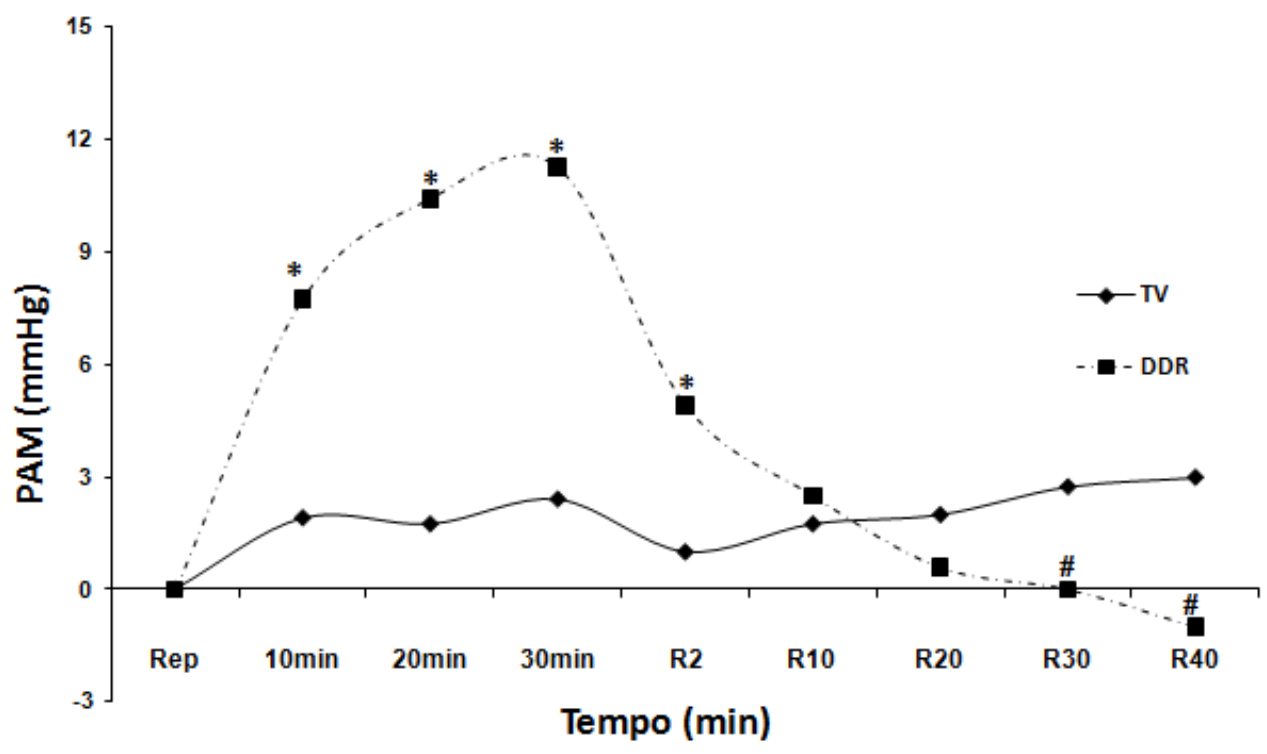

Figura 4. Valores de delta da PAM (mmHg) durante os $30 \mathrm{~min}$ de sessão (10, 20 e $30 \mathrm{~min})$ e durante os minutos 2, 10, 20, 30 e 40 de recuperação (R2, R10, R20, R30 e R40) após as sessões de TV e DDR (Dance Dance Revolution); Rep - repouso; TV ( $F=1,83$ e $p \leq 0,09)$; DDR ( $F=32,68$ e $p \leq 0,01)$. ${ }^{*} p \leq 0,01$ em relação ao repouso; \# $p \leq 0,01$ em relação ao $\mathrm{R} 2$;

\section{Discussão}

O presente estudo comparou as respostas de variáveis cardiovasculares durante e após a prática de vídeo game interativo DDR e televisão em crianças. Os principais resultados foram que, além de elevar o nível de atividade física, o DDR pode promover redução da PA pós-exercício em relação ao controle sem exercício. As variáveis cardiovasculares FC, PAS, PAD e PAM tiveram um aumento durante as atividades com maior exigência física e uma maior redução relativa durante a recuperação.

Já está bem esclarecido na literatura que a atividade física exerce um efeito benéfico sobre $o$ sistema cardiovascular, levando, cronicamente, a diminuição dos valores de $F C$ de repouso (CORNELISSEN, VERHEYDEN, AUBERT, FAGARD, 2010) e agudamente a uma redução da PA após a realização dos exercícios (FORJAZ,
RONDON, NEGRÃO, 2005; CARDOSO Jr. et al., 2010; SIMÕES, SIMÕES, MOREIRA, KUSCHNICK, CAMPBELL, 2010). Tem sido demonstrado em adultos que os benefícios agudos podem persistir por até 24 horas subsequentes a prática (WHELTON, CHIN, XIN, 2002; FAGARD, 2006). Na literatura há poucos estudos analisando estes parâmetros em crianças frente à atividade física.

Observou-se claramente uma tendência à redução da PA pós-exercício em relação ao repouso pré-exercício na sessão DDR, enquanto o contrário foi observado na sessão TV. O pequeno número de voluntários da amostra pode ter contribuído para não se observar o fenômeno da queda significativa da PA após o exercício nessas crianças. As crianças podem ter sido acometidas por um aumento no nível de ansiedade por terem que permanecer sentadas durante os $40 \mathrm{~min}$ após sessões realizadas em 
ambiente de laboratório, resultando assim na não queda significativa da PA, como comumente observada em adultos. Segundo Fonseca, Coelho, Nicolato, Malloy-Diniz, Correa (2009), o ambiente de laboratório pode induzir alteração do estado emocional das crianças podendo contribuir para o aumento da ansiedade e estresse, e consequentemente da PA e FC. A sessão de familiarização foi realizada com intuito de minimizar esse tipo de interferência.

Estes valores de delta de PAS ( $4 \mathrm{mmHg}$ ), após uma única sessão de nível básico do vídeo game ativo DDR, mesmo que não tenham sido significativamente inferiores aos de repouso, se mantidos ao longo do dia pode contribuir para a prevenção do surgimento da HÁ em crianças. Magalhães et al. (2010) destacam a faixa etária pediátrica como alvo principal para implementar e incentivar mudanças no estilo de vida e prevenir o surgimento futuro de DCVs. Isso pode ser obtido reduzindo-se 0 sedentarismo no ambiente doméstico, por exemplo, por meio da introdução de um vídeo game interativo como o DDR.

Estudos populacionais, como o de Pocock et al. (2001) sugerem que uma redução crônica da PA em $2 \mathrm{mmHg}$ pode reduzir o risco de morte súbita em até $6 \%$ e de doenças coronarianas em $4 \%$, em adultos, porém não há informações desse tipo em crianças.

Apesar da queda não significativa da PAS, PAD e PAM durante a recuperação após DDR, podemos sugerir que a regularidade da participação ativa nessa brincadeira, e em níveis mais elevados, pode contribuir para uma redução significativa e manutenção de valores reduzidos de PA em crianças, assim como observado em estudos semelhantes envolvendo exercício físico em adultos (FORJAZ, RONDON, NEGRÃO, 2005;

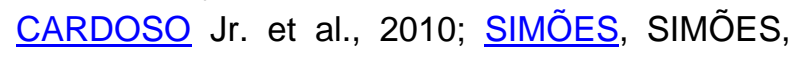
MOREIRA, KUSCHNICK, CAMPBELL, 2010).

Apesar do número amostral pequeno do presente estudo poder ter contribuído para a não observação de diferenças significativas nos parâmetros investigados entre as sessões, as tendências de respostas cardiovasculares entre as sessões, claramente observadas nas figuras 2, 3 e 4 (queda vs elevação), podem ter enorme contribuição clínica na prevenção de eventos cardiovasculares futuros nestas crianças. Em uma metanálise, MacMahon e Rodgers (1993) demonstraram que uma redução de $5 \mathrm{mmHg}$ na PAD foi associada a uma diminuição de pelo menos $34 \%$ de acidente vascular cerebral e $21 \%$ doença coronariana.

O vídeo game interativo é uma opção recente, em que há exigência do potencial físico do praticante, aumento do consumo de oxigênio, da freqüência cardíaca e tem demonstrado ser uma ferramenta interessante na adesão à prática de atividade física (MARK, RHODES, DARREN, WARBURTON, 2008) por parte não só das crianças, mas também de adultos. O vídeo game interativo é uma atividade que não traz todos os benefícios inerentes a brincadeiras ativas realizadas com outras crianças ao ar livre (dimensões psico sócio culturais), porém mostrou-se melhor para a saúde cardiovascular do que quando apenas se assiste TV.

Mealey, Jakicic, Marcus, Mealey (2010) investigaram o gasto energético e a satisfação de crianças obesas (percentil 98) durante a prática do DDR e o caminhar em uma esteira durante $15 \mathrm{~min}$ e não observaram valores estatisticamente diferentes no gasto calórico entre ambos $(78,0 \pm 34,4$ vs $62,30 \pm 15,5 \mathrm{kcal}$, $p=0,093$ ). No entanto, quando analisaram o nível de satisfação durante a prática, as crianças relataram um maior nível de satisfação durante a prática do DDR quando comparado com a caminhada em esteira. Isso pode resultar em uma maior adesão e duração dedicada à prática de atividade física.

Outro fator que consideramos e padronizamos em nosso estudo foi o nível de conhecimento e prática do DDR pelos participantes. Sell, Lillie, Taylor (2008) avaliaram o quanto a experiência com o jogo influenciava as respostas fisiológicas. Os participantes experientes com jogos $(n=12)$ alcançaram maior FC, razão de troca respiratória, consumo de oxigênio e gasto energético total durante a realização do mesmo nível do DDR quando comparados aos não experientes $(n=7)$. Neste caso, a maior experiência na realização da atividade contribuiu para que as crianças atingissem intensidades mais elevadas. Porém essas respostas, assim como as do presente estudo, foram agudas e existe a necessidade de maiores investigações sobre a contribuição desta atividade realizada de forma crônica.

Concluímos que o vídeo game ativo DDR promoveu aumento significativo nas respostas de variáveis cardiovasculares analisadas durante a sua prática, enquanto que após sua realização foi 
observada tendência de redução das mesmas. Por outro lado, na sessão TV a tendência dessas variáveis foi se elevarem. Desta forma, podemos sugerir que o vídeo game ativo é uma alternativa que pode contribuir no combate ao sedentarismo infantil no ambiente doméstico, e na redução da incidência de doenças a ele relacionadas. No entanto, é importante ressaltar que os jogos eletrônicos não devem substituir as brincadeiras tradicionais realizadas com outras crianças ao ar livre junto à natureza.

\section{Referências}

ATKIN, A.J.; GORELY, T.; BIDDLE, S.J.H.; MARSHALL, S.J.; CAMERON, N. Critical hours: physical activity and sedentary behavior of adolescents after school. Pediatric Exercise Science. Springfield, v.20, n.4, p.446-56, 2008. Disponível em:

http://journals.humankinetics.com/pes-backissues/PESVolume20Issue4November/CriticalHou rsPhysicalActivityandSedentaryBehaviorofAdolesc entsAfterSchool Acesso em 15 de jul. 2010.

BAKER, J. L.; OLSEN, L. W.; SORENSEN, T. I. Childhood Body-Mass Index and the Risk of Coronary Heart Disease in Adulthood. The New England Journal of Medicine. Waltham, v.357, n.6, p. 2329-2337, 2007. doi: http://dx.doi.org/10.1056/NEJMoa072515.

CARDOSO JUNIOR, C. G.; GOMIDES, R. S.; QUEIROZ, A. C. C.; PINTO, L. G.; LOBO, F. S.; TINUCCI, T.; MION JUNIOR, D.; FORJAZ, C. L. $M$. Acute and chronic effects of aerobic and resistance exercise on ambulatory blood pressure. Clinics. São Paulo, v. 65, n.3, p.317-325, 2010. doi: http://dx.doi.org/10.1590/S1807$\underline{59322010000300013 .}$.

CARLETTI, L.; RODRIGUES, A. N.; PEREZ, A. J.; VASSALLO, D. V. Blood Pressure Response to Physical Exertion in Adolescents: Influence of Overweight and Obesity. Arquivos Brasileiros de Cardiologia. Rio de Janeiro, v.91, n.1, p. 25-30, 2008. doi: http://dx.doi.org/10.1590/S0066782X2008001300004.

CARVALHO, F. O.; OLIVEIRA, W. A.; CARNEIRO, M. V. O.; MORAES, J. F. V. N.; MOREIRA, S. R.; CAMPBELL, C.S.G.; The Relationship of Body Fat Indicators with Cardiovascular Risk in Adolescents with Low Socioeconomic Status. Journal Exercise Physiology Online, Chicago, v.14, n.3, p.19-29, 2012.

CORNELISSEN, V. A.; VERHEYDEN, B.; AUBERT, A. E.; FAGARD, R. H. Effects of
Aerobic Training Intensity on Resting, Exercise and Post-Exercise Blood Pressure, Heart Rate and Heart-Rate Variability: Aerobic Training and Recovery Blood Pressure. Journal of Human Hypertension. Philadelphia, v.24, p.175-182, 2010. doi: http://dx.doi.org/10.1038/jhh.2009.51.

CORSO, A. C. T.; CALDEIRA, G. V.; FIATES, G. R.; SCHMITZ, B. A. S.; Schmitz, B. A.; RICARDO, G. D.; VASCONCELOS, F. A. G. Fatores comportamentais associados ao sobrepeso e obesidade em escolares do estado de Santa Catarina. Revista Brasileira de Estudos de População (Impresso), Rio de Janeiro, v. 29, p. 117-131, 2012.

FAGARD, R. H. Exercise is Good for Your Blood Pressure: Effects of Endurance Training and Resistance Training. Clinical and Experimental Pharmacology and Physiology. Indianápolis, v. 33, p.853-856, 2006. doi: http://dx.doi.org/10.1111/.1.14401681.2006.04453.x.

FONSECA, F. C. A.; COELHO, R. Z.; NICOLATO,R.; MALLOY-DINIZ, L. F.; CORREA, H. A influência de fatores emocionais sobre a hipertensão arterial. Jornal Brasileiro de Psiquiatria (Impresso), Rio de Janeiro, v. 58, p. 128-134, 2009.

FORJAZ, C. L. M.; RONDON, M. U. P. B.; NEGRÃO,C. E. Efeitos Hipotensores e Simpatolíticos do Exercício Aeróbio na Hipertensão Arterial. Revista Brasileira de Hipertensão. São Paulo, v.12, n. 04, p. 245-250, 2005.

MARTINEZ-GOMEZ D.; TUCKER, J.; HEELAN, K.A.; WELK, G.J.; EISENMANN, J.C. Associations Between Sedentary Behavior and Blood Pressure in Young Children. Archives of Pediatrics \& Adolescent Medicine, v. 163, n. 8, p. 724-730, 2009. doi: http://dx.doi.org/10.1001/archpediatrics.2009.90.

JAGO, R.; BARANOWSKI, T. Non-curricular approaches for increasing physical activity in youth: a review. Preventive Medicine, Maryland, v. 39, p. 157-163, 2004. doi:

http://dx.doi.org/10.1016/i.ypmed.2004.01.014.

LEARY, S.D.; NESS, A. R.; SMITH, G.D.; MATTOCKS, C.; DEERE, K.; BLAIR, S.N. Physical Activity and Blood Pressure in Childhood: Findings from a Population-Based Study. Hypertension, Dallas, v. 51, p. 92-98, 2008. doi: http://dx.doi.org/10.1161/hpertensionaha.107.0990 51.

LUMA, G.B.; SPIOTTA, R.T. Hypertension in children and adolescents. American Family 
Physician, Washington, v. 73, p. 1158-1168, 2006. Disponível em:

http://www.aafp.org/afp/2006/0501/p1558.html

Acesso em: 17 de jun. 2010.

MACMAHON, S.; RODGERS, A. The effects of blood pressure reduction in older patients: an overview of five randomized controlled trials in elderly hypertensives. Clinical and Experimental Hypertension, New Jersey, v. 15, p. 967-78, 1993. doi: http://dx.doi.org/10.1016/S01406736(94)90696-3.

MARK, R.; RHODES, R.; DARREN, E. R.; WARBURTON, S. B. Interactive Video Games and Physical Activity: A Review of the Literature and Future Directions. Health \&Fitness Journal of Canada, Canada, v. 1, n. 1, p. 14-24, 2008. Disponível em: http://www.healthandfitnessjournalofcanada.com/i ndex.php/html/article/view/5. Acesso em: 15 de jun. 2010.

MEALEY, L.; JAKICIC, J. M. M. D.; MARCUS; MEALEY, A. D. The Effect Of Dance Dance Revolution On Energy Expenditure And Enjoyment In Severely Overweight Children Compared To Alternate Forms Of Activity. The Journal of Strength \& Conditioning Research, Pennsylvania, v. 24, p. 1-10, 2010. doi: http://dx.doi.org/10.1097/01.JSC.0000367173.791 49.ef.

National High Blood Pressure Education Program Working Group On High Blood Pressure In Children And Adolescents. The Fourth Report on the Diagnosis, Evaluation, and Treatment of High Blood Pressure in Children and Adolescents.

Pediatrics, 2004. doi: http://dx.doi.org/114: 555$\underline{576 .}$

OBARZANEK, E.; WU, C.O.; CUTLER, J. A.; KAVEY, R. E.; PEARSON, G. D.; DANIELS, S. R. Prevalence and Incidence of Hypertension in Adolescent Girls. The Journal of Pediatrics, New York, v. 10, p. 7-5, 2010. doi:

http://dx.doi.org/10.1016/j.jpeds.2010.03.032.

PELEGRINI, A.; SILVA, R. C. R.; PETROSKI, E. L. Relação entre o tempo em frente à TV e o gasto calórico em adolescentes com diferentes percentuais de gordura corporal. Revista

Brasileira de Cineantropometria \&

Desempenho Humano, Florianópolis, v. 10, p. 81-84, 2008.

POCOCK, S. J.; MCCORMACK, V.; GUEYFFIER, F.; BOUTITIE, F.; FAGARD, R. H.; BOISSEL, J-P. A score for predicting risk of death from cardiovascular disease in adults with raised blood pressure, based on individual patient data from randomised controlled trials. British Medical
Journal, London, v. 323, p. 75-81, 2001. doi: http://dx.doi.org/10.1136/bmj.323.7304.75.

ROBINSON, T. N.; Reducing children's television viewing to prevent obesity. Journal Of the American Medical Association, Chicago, v. 282, p. 1561-1567, 1999. doi: http://dx.doi.org/10.1001/jama.282.16.1561.

SELL, K.; LILLIE, I.; TAYLOR, J. Energy expenditure during physically interactive video game playing in male college students with different playing experience. The Journal of American College Health, Linthicum, v. 56, n. 05, p. 505-511, 2008. doi: http://dx.doi.org/10.1001/jama.282.16.1561.

SLAUGHTER, M. H.; LOHMAN, T. G.; BOILEAU, R. A.; HORSWILL, C. A.; STILLMAN, R. J.; VAN LOAN, M.D. Skinfold equations for estimation of body fatness in children and youth. Human Biology, Paris, v. 60, p. 709-23, 1988.

SIMÕES, G. C.; SIMÕES, H.G.; MOREIRA, S. R.; KUSCHNICK, M. R.; CAMPBELL, C. S. G. PostResistance Exercise Blood Pressure Reduction is Influenced by Exercise Intensity in Healthy Individuals and in those with Type-2 Diabetes. The Journal of Strength and Conditioning Research, Pennsylvania, v. 24, p. 1277-1284, 2010. doi:

http://dx.doi.org/10.1519/JSC.0b013e3181d67488.

SYME, C.; ABRAHAMOWICZ, M.; PERRON, M.; RICHER, L.; VEILLETTE, S.; XIAO, Y.; GAUDET, D.; PAUS, P.; PAUSOVA, Z. Sex Differences in Blood Pressure and Its Relationship to Body Composition and Metabolism in Adolescence.

Archives of Pediatrics \& Adolescent Medicine, Chicago, v. 163, n. 9, p. 818-825, 2009. doi: http://dx.doi.org/10.1001/archpediatrics.2009.92.

Task Force on Blood Pressure Control in Children. Update on the 1987 Task Force Report on High Blood Pressure in Children and Adolescents: a Working Group Report from the National High Blood Pressure Education Program National High Blood Pressure Education Program Working Group on Hypertension Control in Children and Adolescents. Pediatrics, v. 98, p. 649-58, 1996.

TROIANO, R. P.; BERRIGAN, D.; DODD, K.W.; MÂSSE, L.C.; TILERT, T.; MCDOWELL, M. Physical Activity in the United States Measured by Accelerometer. Medicine \& Science in Sports \& Exercise, Indianapolis, v. 40, p. 181-188, 2008. doi:

http://dx.doi.org/10.1249/MSS.0b013e31817057da

WANG, X.; PERRY, A.C. Metabolic and physiologic responses to video game play in 7- to 10 -year old boys. Archives of Pediatrics \& Adolescent Medicine. Chicago, USA, v. 160, p. 
411-415, 2006. doi:

http://dx.doi.org/10.1001/archpedi.160.4.411.

WHELTON, S. P.; CHIN, A.; XIN, X.; HE, J. Effect of Aerobic Exercise on Blood Pressure: a Metaanalysis of Randomized, Controlled Trials. Annals of Internal Medicine. Philadelphia - USA, v.136, p. 493-503, 2002.

http://dx.doi.org/10.1097/HJR.0b013e3280128bbb

WHO (World Health Organization). Global recommendations on physical activity for health. 2010.

Agradecimento: Agradecimento especial a todos os voluntários da pesquisa e a seus pais e/ou responsáveis por permitir que eles participassem da pesquisa.

Agências de Financiamento: Ao Conselho Nacional de Desenvolvimento Científico e Tecnológico - CNPq e a Coordenação de Aperfeiçoamento de Pessoal de Nível Superior CAPES pelas bolsas outorgadas aos pesquisadores.

\section{Endereço:}

Ferdinando Oliveira Carvalho

Rua José de Sá Maniçoba, S/N - Centro

Petrolina PE Brasil

56304-917

Telefone: (87) 2101-6856

e-mail: ferdinando.carvalho@univasf.edu.br

Recebido em: 6 de setembro de 2010.

Aceito em: 17 de abril de 2013.

\section{(c) (i)}

Motriz. Revista de Educação Física. UNESP, Rio Claro, SP, Brasil - elSSN: 1980-6574 - está licenciada sob Creative Commons - Atribuição 3.0 